\title{
Opioid use and abuse following video-assisted thoracic surgery (VATS) or thoracotomy lung cancer surgery
}

\author{
Tamar B. Nobel, Prasad S. Adusumilli, Daniela Molena \\ Department of Surgery, Memorial Sloan Kettering Cancer Center, New York, NY, USA \\ Correspondence to: Daniela Molena, MD. Associate Attending, Department of Surgery, 1275 York Avenue, New York, NY 10065, USA. \\ Email: molenad@mskcc.org. \\ Provenance: This is an invited article commissioned by the Section Editor Hengrui Liang (Department of Thoracic Surgery, Guangzhou Medical \\ University, Guangzhou, China). \\ Comment on: Tuminello S, Schwartz RM, Liu B, et al. Opioid Use After Open Resection or Video-Assisted Thoracoscopic Surgery for Early-Stage \\ Lung Cancer. JAMA Oncol 2018;4:1611-3.
}

Submitted Apr 26, 2019. Accepted for publication May 20, 2019.

doi: $10.21037 /$ tlcr.2019.05.14

View this article at: http://dx.doi.org/10.21037/tlcr.2019.05.14

\begin{abstract}
Although opioids are frequently prescribed in the postoperative setting, the call for increased opioid stewardship among surgeons has only recently become more urgent. Recent studies have shown that opioids are overprescribed postoperatively $(1,2)$. Given that the risk of chronic opioid use and consequently, misuse and abuse, has been demonstrated to increase with longer duration and higher dosage prescriptions, it is time for surgeons to have a proactive role in mitigating the opioid crisis $(3,4)$. Most of the current literature to date on postoperative opioid prescribing has focused on general, gynecologic, and orthopedic surgery $(1-3,5,6)$. Opioid prescribing patterns and implications for thoracic surgery remain mostly undefined.
\end{abstract}

\section{Post-thoracic surgery pain and analgesia}

Postoperative pain after thoracic surgery is uniquely challenging both in the acute and long-term setting. The observed high rate of pain has been attributed to rib spread and intercostal nerve damage intraoperatively. In the immediate postoperative setting, inadequate postoperative pain control may increase splinting, inadequate coughing and impaired respiratory mechanics resulting in increased hypoxia, atelectasis and infection (7). Furthermore, chronic pain post-thoracotomy has been reported in up to $80 \%$ of patients within 6 months of surgery and $30-50 \%$ up to 5 years postoperatively (8-11). Given that acute postoperative pain after thoracic surgery has been associated with increased likelihood of chronic pain, aggressive perioperative pain control is very important (12). A multimodal, multidisciplinary approach involving regional and systemic perioperative analgesia has become standard to mitigate postoperative pain.

Regional pain control may be achieved by several mechanisms. Preoperative placement of a thoracic epidural with infusion of local anesthetic and/or opioids is the current standard, used intraoperatively and for postoperative patientcontrolled analgesia. However, complications of thoracic epidural, including hypotension, epidural hematoma and urinary retention, as well as failure rates of $14-30 \%$, have made paravertebral blockade an attractive alternative with similar analgesia achieved and a lower minor complication rate (11).

In the current era of video-assisted thoracic surgery (VATS) and associated smaller incisions without need for rib spreading, alternative methods of local pain control have been utilized to avoid the potential associated complications with thoracic epidural. Intracostal nerve block may be performed under direct vision intraoperatively (13). Alternatively, intercostal catheter placement has also been described as an alternative mechanism for postoperative continuous analgesic infusion that may allow for earlier hospital discharge than traditional epidural $(14,15)$. Furthermore, as compared to traditional VATS, the advent of uniportal VATS may have the important advantage 
of being even less traumatic and therefore result in less pain postoperatively (16). Finally, there have been several reports from Asia regarding use of spontaneous ventilation anesthesia for thoracoscopic procedures, with the potential benefit of quicker postoperative recovery and fewer side effects than general anesthesia (17). Such an approach may involve using intravenous anesthesia alone in combination with local or regional anesthetic, and has been demonstrated to have only a $1 \%$ conversion rate to tracheal intubation in a recent series of 240 cases (18).

Postoperatively, systemic opioid analgesics are given as an adjunct and become the mainstay of inpatient treatment once invasive catheters are removed, as well as upon hospital discharge (13). In the era of enhanced recovery after surgery (ERAS) pathways, there has been increasing emphasis on concurrent use of non-opioid medications to reduce narcotic use. Gabapentin has been effectively used to treat peripheral neuropathy and has shown promising opioid-sparing effects as an adjunct in the postoperative setting; however, data on use in thoracic surgery is limited given that ERAS has only more recently been adopted for these procedures (19). It is an important component of the ERAS pathway at our institution.

There is some evidence to suggest that an "opioid paradox" exists whereby increased intraoperative opioid use may result in a greater amount of opioids required postoperatively (20). New data supports this finding, demonstrating that opioid-free anesthesia (OFA) may be associated with significant reductions in postoperative opioid use (21). It is possible that this approach has an important role within the ERAS pathway, as demonstrated by a recent study in neurosurgery that demonstrated that the total amount of opioids received perioperatively by traditional anesthesia patients was $16 \times$ higher than OFA patients (22). It remains to be determined whether this approach may be beneficial in thoracic surgery.

\section{The impact of operative approach on postoperative pain}

VATS has become the standard approach to lung cancer resection. In addition to shorter length of stay and lower complication rates, another suggested benefit of the VATS approach includes reduced pain postoperatively (23). Although it is logical that patients would report less pain after undergoing a less traumatic procedure, the painreducing benefits of the VATS approach have not been consistently demonstrated.

Two early studies from the 1990s that evaluated pain and associated pulmonary function measures demonstrated the superiority of the VATS approach in the immediate postoperative period $(24,25)$. However, more recent studies have had mixed findings. In a recent randomized trial by Bendixen et al., 206 patients were randomized to lobectomy via either VATS or anterolateral thoracotomy (23). Comparison of pain reported in the 12 months after surgery demonstrated a marginally lower rate of moderate-to-severe pain in the VATS group, and furthermore, no significant difference in severe pain was identified. Similarly, a 2014 prospective series published by Rizk et al. from our own institution demonstrated similar pain scores between VATS and thoracotomy immediately postoperatively as well as 4, 8 and 12 months after surgery (26). Importantly, neither the Bendixen nor the Rizk study demonstrated a significant difference in global quality-of-life $(23,26)$.

Obuchi et al. provide a potential explanation for the observed lack of difference in their comparison of postoperative pain for VATS versus thoracotomy patients (27). VATS patients without epidural had significantly more pain than VATS patients that did not receive an epidural; however, when comparing VATS and thoracotomy patients that all received epidural, there was no difference between groups. Aggressive postoperative pain control in the acute setting may mask inherent differences in acute postoperative pain between groups.

Assessment of long-term outcomes is limited; however, a 2011 survey administered to VATS and thoracotomy patients in Scandinavia at a median of 22 months followup postoperatively failed to demonstrate consistent differences in the prevalence or anatomical distribution of pain, sensory changes or effect of pain on daily activities (28). Additionally, a similar rate of VATS and thoracotomy patients reported taking opioids for pain at time of survey administration.

There is a need for further randomized studies to characterize the true nature of the benefits of the VATS approach on postoperative pain; however, review of the literature appears to suggest that any benefits may be predominantly experienced in the acute postoperative setting.

\section{Operative approach and chronic opioid use}

In the setting of the observed relationships between postoperative pain control and chronic pain post thoracic 
surgery as well as that between acute postoperative opioid use and subsequent chronic use, Tuminello et al. present an interesting question in their article entitled, "Opioid Use After Open Resection or Video-Assisted Thoracoscopic Surgery for Early-Stage Lung Cancer" (29). In this series, the authors compared incidence of long-term opioid use between open and VATS lung surgery in non-small cell lung cancer patients identified in the Surveillance, Epidemiology, and End Results (SEER) Medicare-linked database (29). In order to exclude the possibility of increased pain sensitization resulting from chronic pain conditions, tolerance from chronic opioid use as well as cancer related pain, the authors included only opioid-naïve patients and early stage patients in this study.

The authors confirmed that chronic postoperative use was higher among patients that underwent open resection as compared to VATS $(19 \%$ vs. $12 \%, \mathrm{P}<0.0001)$. VATS patients also had a significantly lower rate of filling an opioid prescription within 90 days postoperatively and filled fewer opioid prescriptions overall than open resection patients. After propensity matching, the analysis confirmed that the risk of chronic opioid use was still significantly lower among VATS patients (OR 0.52, 95\% CI, 0.36-0.75). The authors concluded that surgical invasiveness may have a role in the odds of becoming a long-term opioid user after surgery.

\section{Study message}

Tuminello et al. should be commended for addressing a very timely and important issue in thoracic surgery. Postoperative pain has important implications not only for patient qualityof-life, but also for the potential for chronic opioid abuse. Interestingly, this study demonstrated a chronic use rate of $16 \%$ among all patients; as this is significantly higher than that reported in the general surgery literature (up to 7\%), this may demonstrate the particularly painful nature of thoracic surgery (3). As such, thoracic surgeons in particular have an important challenge in the current opioid crisis.

The results of the current study demonstrate a strong relationship between thoracotomy and increased opioid medication prescribed at $0-3$ and $3-6$ months. If opioid prescriptions are to be considered as a proxy for postoperative pain and pain management after surgery, this is in sharp contrast to the previously described studies. There are several reasons for the difference in findings. First, the current study utilized a national database of nearly 4,000 patients from a variety of hospitals. In comparison, the Rizk and Bendixen studies were performed at single academic institutions and as such, there is a possibility for selection bias. Second, because of the limitations of the SEER database, Tuminello et al. did not include number of pills consumed. It is important not to confuse the number of prescriptions written with the number of pills consumed, which may be a stronger surrogate for pain. Although the current study does not allow for any conclusive assessment of differences in postoperative pain, the results do suggest that thoracotomy may have worse pain postoperatively.

\section{Study limitations}

We believe the findings of this study should be interpreted with caution for several methodologic reasons. The authors do not present data on the amount of medication prescribed postoperatively. Furthermore, the authors do not provide data on how many patients filled a prescription initially postoperatively and required a subsequent refill within 90 days. It has been demonstrated that prescribing a higher dose or quantity of pills in the postoperative setting is associated with increased likelihood of chronic use and misuse $(4,30)$. A recent clinical trial randomized patients undergoing hand surgery to 10 or 30 pills, and demonstrated a lower rate of total pills used and persistent opioid use among those that received fewer pills without compromising satisfaction with pain controls or need for increased refills (31). Without knowing how much medication was prescribed, it is not possible to determine whether chronic opioid use is the result of increased pain or abuse as a consequence of initial overprescribing. In the current ERAS era, VATS patients are managed in a multimodal, opioid-sparing approach so they may receive fewer opioids after surgery to begin with. In support of this, the authors did demonstrate that the risk of chronic use decreased with more recent year of diagnosis.

In addition to the role of ERAS, there are other factors that may contribute to selection bias that may influence the observed results. There were more patients in the higher income quartiles that developed long-term opioid use. Such patients may be more likely to go to high-quality centers that use VATS and adhere to ERAS or may be better informed regarding the consequences of opioid medication use.

Finally, by using the SEER-Medicare population the cohort is limited to patients $65+$. Given that younger age has been associated with chronic use elsewhere, it is possible that the true magnitude of this relationship is masked by including only older patients (3). 


\section{Summary}

The current study addresses an important issue facing surgeons today. Further research should be performed to better characterize the unique aspects of thoracic surgery that result in high rates of chronic opioid use to enable early identification of at-risk patients, including assessment of postoperative analgesia approaches and development of guidelines for postoperative opioid prescribing. Furthermore, it is important that surgeons set expectations with patients for postoperative pain and provide education on postoperative opioid use.

\section{Acknowledgments}

None.

\section{Footnote}

Conflicts of Interest: The authors have no conflicts of interest to declare.

\section{References}

1. Howard R, Fry B, Gunaseelan V, et al. Association of Opioid Prescribing With Opioid Consumption After Surgery in Michigan. JAMA Surg 2019;154:e184234.

2. Hill MV, McMahon ML, Stucke RS, et al. Wide Variation and Excessive Dosage of Opioid Prescriptions for Common General Surgical Procedures. Ann Surg 2017;265:709-14.

3. Brummett CM, Waljee JF, Goesling J, et al. New Persistent Opioid Use After Minor and Major Surgical Procedures in US Adults. JAMA Surg 2017;152:e170504.

4. Brat GA, Agniel D, Beam A, et al. Postsurgical prescriptions for opioid naive patients and association with overdose and misuse: retrospective cohort study. BMJ 2018;360:j5790.

5. Nobel TB, Zaveri S, Khetan P, et al. Temporal trends in opioid prescribing for common general surgical procedures in the opioid crisis era. Am J Surg 2019;217:613-7.

6. Hill MV, Stucke RS, McMahon ML, et al. An Educational Intervention Decreases Opioid Prescribing After General Surgical Operations. Ann Surg 2018;267:468-72.

7. Goto T. What is the best pain control after thoracic surgery? J Thorac Dis 2018;10:1335-8.

8. Kinney MA, Hooten WM, Cassivi SD, et al. Chronic Postthoracotomy Pain and Health-Related Quality of Life.
Ann Thorac Surg 2012;93:1242-7.

9. Guastella V, Mick G, Soriano C, et al. A prospective study of neuropathic pain induced by thoracotomy: incidence, clinical description, and diagnosis. Pain 2011;152:74-81.

10. Pluijms WA, Steegers MA, Verhagen AF, et al. Chronic post-thoracotomy pain: a retrospective study. Acta Anaesthesiol Scand 2006;50:804-8.

11. Yeung JH, Gates S, Naidu BV, et al. Paravertebral block versus thoracic epidural for patients undergoing thoracotomy. Cochrane Database Syst Rev 2016;2:CD009121.

12. Katz J, Jackson M, Kavanagh BP, et al. Acute pain after thoracic surgery predicts long-term post-thoracotomy pain. Clin J Pain 1996;12:50-5.

13. Gottschalk A, Cohen SP, Yang S, et al. Preventing and treating pain after thoracic surgery. Anesthesiology 2006;104:594-600.

14. Wildgaard K, Petersen RH, Hansen HJ, et al. Multimodal analgesic treatment in video-assisted thoracic surgery lobectomy using an intraoperative intercostal catheter. Eur J Cardiothorac Surg 2012;41:1072-7.

15. Gebhardt R, Mehran RJ, Soliz J, et al. Epidural versus ON-Q local anesthetic-infiltrating catheter for postthoracotomy pain control. J Cardiothorac Vasc Anesth 2013;27:423-6.

16. Wang $\mathrm{L}$, Liu $\mathrm{D}, \mathrm{Lu} \mathrm{J}$, et al. The feasibility and advantage of uniportal video-assisted thoracoscopic surgery (VATS) in pulmonary lobectomy. BMC Cancer 2017;17:75.

17. Ng CS, Ho JY, Zhao ZR. Spontaneous ventilation anaesthesia: the perfect match for thoracoscopic bullectomy? Eur J Cardiothorac Surg 2016;50:933.

18. Guo Z, Yin W, Wang W, et al. Spontaneous ventilation anaesthesia: total intravenous anaesthesia with local anaesthesia or thoracic epidural anaesthesia for thoracoscopic bullectomy. Eur J Cardiothorac Surg 2016;50:927-32.

19. Grosen K, Drewes AM, Højsgaard A, et al. Perioperative gabapentin for the prevention of persistent pain after thoracotomy: a randomized controlled trial. Eur J Cardiothorac Surg 2014;46:76-85.

20. Koepke EJ, Manning EL, Miller TE, et al. The rising tide of opioid use and abuse: the role of the anesthesiologist. Perioper Med (Lond) 2018;7:16.

21. Samuels S, Abou-Samra A, Dalvi P, et al. Opioid-free Anesthesia Results in Reduced Postoperative Opioid Consumption. J Clin Anesth Pain Med 2017;1(2).

22. Soffin EM, Wetmore DS, Beckman JD, et al. Opioidfree anesthesia within an enhanced recovery after surgery 
pathway for minimally invasive lumbar spine surgery: a retrospective matched cohort study. Neurosurg Focus 2019;46:E8.

23. Bendixen M, Jorgensen OD, Kronborg C, et al. Postoperative pain and quality of life after lobectomy via video-assisted thoracoscopic surgery or anterolateral thoracotomy for early stage lung cancer: a randomised controlled trial. Lancet Oncol 2016;17:836-44.

24. Tschernko EM, Hofer S, Bieglmayer C, et al. Early postoperative stress: video-assisted wedge resection/ lobectomy vs conventional axillary thoracotomy. Chest 1996;109:1636-42.

25. Landreneau RJ, Hazelrigg SR, Mack MJ, et al. Postoperative pain-related morbidity: Video-assisted thoracic surgery versus thoracotomy. Ann Thorac Surg 1993;56:1285-9.

26. Rizk NP, Ghanie A, Hsu M, et al. A prospective trial comparing pain and quality of life measures after anatomic lung resection using thoracoscopy or thoracotomy. Ann Thorac Surg 2014;98:1160-6.

Cite this article as: Nobel TB, Adusumilli PS, Molena D. Opioid use and abuse following video-assisted thoracic surgery (VATS) or thoracotomy lung cancer surgery. Transl Lung Cancer Res 2019;8(Suppl 4):S373-S377. doi: 10.21037/ tlcr.2019.05.14
27. Obuchi T, Yoshida Y, Moroga T, et al. Postoperative pain in thoracic surgery: re-evaluating the benefits of VATS when coupled with epidural analgesia. J Thorac Dis 2017;9:4347-52.

28. Wildgaard K, Ravin J, Nikolajsen L, et al. Consequences of persistent pain after lung cancer surgery: a nationwide questionnaire study. Acta Anaesthesiol Scand 2011;55:60-8.

29. Tuminello S, Schwartz RM, Liu B, et al. Opioid Use After Open Resection or Video-Assisted Thoracoscopic Surgery for Early-Stage Lung Cancer. JAMA Oncol 2018;4:1611-3.

30. Zaveri S, Nobel TB, Khetan P, et al. Risk of Chronic Opioid Use in Opioid-Naïve and Non-Naïve Patients after Ambulatory Surgery. J Gastrointest Surg 2019. [Epub ahead of print].

31. Gaddis A, Dowlati E, Apel PJ, et al. Effect of Prescription Size on Opioid Use and Patient Satisfaction After Minor Hand Surgery: A Randomized Clinical Trial. Ann Surg 2019. doi: 10.1097/SLA.0000000000003127. 